\title{
On quadratic matrix equations with infinite size coefficients encountered in QBD stochastic processes
}

\author{
D.A. Bini ${ }^{1}$, S. Massei ${ }^{2}$, B. Meini ${ }^{1}$, L. Robol ${ }^{3}$ \\ ${ }^{1}$ Dipartimento di Matematica, Università di Pisa, Italy \\ ${ }^{2}$ EPF Lausanne, Switzerland \\ ${ }^{3}$ ISTI-CNR, Pisa, Italy
}

\begin{abstract}
SUMMARY
Matrix equations of the kind $A_{1} X^{2}+A_{0} X+A_{-1}=X$, where both the matrix coefficients and the unknown are semi-infinite matrices belonging to a Banach algebra, are considered. These equations, where coefficients are quasi-Toeplitz matrices, are encountered in certain Quasi-Birth-Death (QBD) processes as the tandem Jackson queue or in any other process which can be modelled as a reflecting random walk in the quarter plane. We provide a numerical framework for approximating the minimal nonnegative solution of these equations which relies on semi-infinite quasi-Toeplitz matrix arithmetic. In particular, we show that the algorithm of Cyclic Reduction can be effectively applied and can approximate the infinite dimensional solutions with quadratic convergence at a cost which is comparable to that of the finite case. This way, we may compute a finite approximation of the sought solution, as well as of the invariant probability measure of the associated QBD process, within a given accuracy. Numerical experiments, performed on a collection of benchmarks, confirm the theoretical analysis. Copyright (C) 0000 John Wiley \& Sons, Ltd.
\end{abstract}

Received ...

\section{INTRODUCTION}

In the analysis of certain queueing processes like the tandem Jackson queue [1] or bi-dimensional random walks in the quarter of plane [2,3], one has to find the invariant probability vector $\pi$ of a stochastic process with a discrete two-dimensional state space. The two coordinates of the latter, usually called level and phase, are assumed countably infinite. Typically, the transitions from a state are limited to a subset of adjacent states and are homogeneous in time. This makes the transition probability matrix $P$ semi-infinite, block-tridiagonal, almost block-Toeplitz with semiinfinite almost-Toeplitz blocks. More precisely, $P$ is a row-stochastic matrix of the form

$$
P=\left[\begin{array}{ccccc}
B_{0} & B_{1} & & & \\
A_{-1} & A_{0} & A_{1} & & \\
& A_{-1} & A_{0} & A_{1} & \\
& & \ddots & \ddots & \ddots
\end{array}\right],
$$

*Correspondence to: E-mail: dario.bini@unipi.it 
where all the blocks $B_{0}, B_{1}$ and $A_{i}, i=-1,0,1$, are semi-infinite tridiagonal matrices having a quasi-Toeplitz structure, that is,

$$
A_{i}=\left[\begin{array}{ccccc}
b_{0}^{(i)} & b_{1}^{(i)} & & & \\
a_{-1}^{(i)} & a_{0}^{(i)} & a_{1}^{(i)} & & \\
& a_{-1}^{(i)} & a_{0}^{(i)} & a_{1}^{(i)} & \\
& & \ddots & \ddots & \ddots
\end{array}\right], \quad B_{j}=\left[\begin{array}{ccccc}
\widehat{b}_{0}^{(j)} & \widehat{b}_{1}^{(j)} & & & \\
\widehat{a}_{-1}^{(j)} & \widehat{a}_{0}^{(j)} & \widehat{a}_{1}^{(j)} & & \\
& \widehat{a}_{-1}^{(j)} & \widehat{a}_{0}^{(j)} & \widehat{a}_{1}^{(j)} & \\
& & \ddots & \ddots & \ddots
\end{array}\right],
$$

for $i=-1,0,1$ and $j=0,1$.

Observe that, except for the two entries in the first row, both the block matrix (1) and each block $A_{i}, B_{j}$ in (2) have the same (block) entries equally repeated along each diagonal. Matrices with repeated (block) entries along the (block) diagonals are known as (block) Toeplitz matrices.

Stochastic processes with a block-tridiagonal almost block-Toeplitz transition matrix, as the matrix $P$ of (1), are known as Quasi-Birth-Death processes, in short, QBD. If the blocks $A_{i}$, $i=-1,0,1$, and $B_{i}, i=0,1$ are semi-infinite matrices, the QBD is said to have an infinite number of phases.

The steady state vector $\pi$, if it exists, is the nonnegative row vector with sum of its entries equal to 1 , such that $\pi P=\pi$. By partitioning $\pi$ in the form $\pi=\left[\pi_{0}, \pi_{1}, \ldots\right]$ according to the block structure of $P$, one finds that [4] the following matrix geometric relation holds

$$
\pi_{n}=\pi_{0} R^{n}, \quad i \in \mathbb{N},
$$

where $R$ is the minimal non negative solution of the matrix equation

$$
X=X^{2} A_{-1}+X A_{0}+A_{1} .
$$

Moreover, the vector $\pi_{0}$ is such that $\pi_{0}\left(B_{0}+B_{1} G\right)=\pi_{0}$, where $G$ is the minimal nonnegative solution of the matrix equation

$$
X=A_{-1}+A_{0} X+A_{1} X^{2} .
$$

These solutions $R$ and $G$ always exist. Here, we are interested in the important case from the applications where the process is positive recurrent. In this case, the vector $\pi$ exists and is unique, the matrix $G$ is stochastic, i.e., $G \mathbf{1}=\mathbf{1}$ where $\mathbf{1}$ is the vector of all ones, and the series $\sum_{i=0}^{\infty} R^{i}$ is convergent [5].

In this paper, we consider matrix equations of the kinds (3) and (4) where the matrix coefficients are semi-infinite matrices belonging to a Banach algebra. A case of interest is the one in which matrices are quasi-Toeplitz. A quasi-Toeplitz matrix has the form $T(a)+F$, where $T(a)=\left(t_{i, j}\right)$ is the Toeplitz matrix associated with the symbol $a(z)=\sum_{i \in \mathbb{Z}} a_{i} z^{i}$, that is, $t_{i, j}=a_{j-i}$, and $F=\left(f_{i, j}\right)$ is such that $\sum_{i, j \in \mathbb{Z}}\left|f_{i, j}\right|<+\infty$. Observe that the matrices in (2) are quasi-Toeplitz.

In the case where the matrices $A_{i}, i=-1,0,1$, belong to a Banach algebra $\mathcal{B}$ we relate the solutions of equations (3) and (4) to the factorization of the matrix Laurent polynomial $\varphi(z)=$ $A_{-1} z^{-1}+\left(A_{0}-I\right)+A_{1} z$ and to the coefficients of the Laurent series representing its inverse. These properties allow one to extend to the infinite dimensional case the convergence results of the Cyclic Reduction (CR) algorithm, which is an efficient numerical method designed to solve equations (3) and (4) in the finite dimensional case [6], [7].

If $\mathcal{B}$ is the Banach algebra of quasi-Toeplitz matrices, the matrix sequences generated by Cyclic Reduction are quasi-Toeplitz and can be represented, up to any arbitrary precision, with a finite number of parameters. This leads to an efficient computation of $R$ and $G$. Moreover, we provide a way to compute the components of the infinite vectors $\pi_{i}, i=0,1, \ldots$ which are greater than a given tolerance. We present numerical experiments which validate the algorithmic and theoretical results obtained in our analysis.

The paper is organized as follows. Section 2 contains some preliminary tools needed throughout the paper. More specifically, in Section 2.1 we recall the main properties of QBD processes, in 
Section 2.2 we recall the class $\mathcal{Q T}$ of quasi-Toeplitz matrices introduced in [8], while in Section 2.3 we deal with the CR algorithm for solving equations (3), (4) in the finite dimensional case. In Section 3 we consider the case where $A_{i} \in \mathcal{B}, i=-1,0,1$ and we relate the solutions $R$ and $G$ to a suitable factorization of $\varphi(z)=A_{-1} z^{-1}+\left(A_{0}-I\right)+A_{1} z$ and to the coefficients of the Laurent series representing its inverse. In Section 4 we provide some convergence properties of CR in the general case $A_{i} \in \mathcal{B}, i=-1,0,1$. In Section 5 we deal with the specific case where equations (3) and (4) come from a QBD where the blocks $A_{i}$ are quasi-Toeplitz. The computation of the vector $\pi$ is treated in Section 6, while numerical experiments are presented in Section 7.

\section{PRELIMINARIES}

We recall some properties of QBDs, quasi-Toeplitz matrices and the algorithm of cyclic reduction.

\subsection{QBD processes}

The minimal nonnegative solutions $R$ and $G$ of the equations (3) and (4), respectively, induce a factorization of the Laurent matrix polynomial

$$
\varphi(z)=z^{-1} A_{-1}+\left(A_{0}-I\right)+z A_{1}
$$

as stated by the following result, which resumes properties proved in [4, Section 6.2], [9] and [10].

\section{Theorem 1}

Assume that the matrix $P$ of (1) is row stochastic. Then there exist minimal nonnegative solutions $R, G$ of the equations (3) and (4) and the factorization

$$
\varphi(z)=(I-z R)(U-I)\left(I-z^{-1} G\right)
$$

holds, where $U=A_{0}+A_{1} G=A_{0}+R A_{-1}$ is a nonnegative matrix such that $U \mathbf{1} \leq \mathbf{1}$ and $\sum_{i=0}^{\infty} U^{i}<\infty$ elementwise.

Assume that the Markov chain having transition matrix $P$ of (1) is irreducible. According to $[5,11]$, such Markov chain is positive recurrent if and only if

$$
\sum_{i=0}^{\infty} R^{i} \mathbf{1}<\infty
$$

elementwise and the system

$$
\pi_{0}=\pi_{0}\left(B_{0}+B_{1} G\right), \quad \pi_{0} \sum_{i=0}^{\infty} R^{i} \mathbf{1}=1
$$

has a nonnegative solution. Moreover, the row vector $\pi$ such that $\pi P=\pi, \pi \mathbf{1}=1$, partitioned as $\pi=\left[\pi_{0}, \pi_{1}, \pi_{2}, \ldots\right]$ according to the structure of $P$, can be obtained by the formula $\pi_{k}=\pi_{k-1} R$, for $k \geq 1$. In the positive recurrent case the matrix $G$ is stochastic, i.e., $G \mathbf{1}=\mathbf{1}$.

Observe that, if the blocks forming $P$ are finite dimensional, so that $R$ is finite dimensional, equation (5) is equivalent to $\rho(R)<1$, where $\rho(\cdot)$ denotes the spectral radius. Moreover, if $\rho(R)<$ 1 , the system (6) has always a solution. In the infinite dimensional case it is convenient to define

$$
c(R)=\sup \left\{z \in \mathbb{R}, z \geq 0: \sum_{i=0}^{\infty} z^{i} R^{i}<\infty\right\}
$$

where inequality is intended elementwise. In view of (5), if $c(R)>1$ and the system (6) has a nonnegative solution, then the QBD is positive recurrent. In the case where the blocks $A_{i}$, 
$i=-1,0,1$, are infinite-dimensional, tridiagonal and almost Toeplitz, necessary and sufficient conditions for $c(R)>1$ are given in [9], [2].

In the sequel of the paper, since we are dealing with infinite-dimensional matrices, we assume that $c(R)>1$ and that the system (6) has a nonnegative solution.

Under this assumption, there exists a real number $0<\alpha<1$ such that both the series $\sum_{i=0}^{\infty} \alpha^{-i} R^{i}$ and $\sum_{i=0}^{\infty} \alpha^{i} G^{i}$ are bounded elementwise. Indeed, for the first series it is sufficient to choose $0<$ $\alpha^{-1}<c(R)$, while for the second series, since $G \mathbf{1}=\mathbf{1}$, it is sufficient to choose $0<\alpha<1$. Thus we get the condition $c(R)^{-1}<\alpha<1$. With this choice of $\alpha$, since $\varphi\left(\alpha^{-1} z\right)=\left(I-z \alpha^{-1} R\right)(U-$ $I)\left(I-z^{-1} \alpha G\right)$, then the matrix $\varphi\left(\alpha^{-1} z\right)$ is invertible for any $z$ belonging to an annulus containing the unit circle.

\subsection{Quasi-Toeplitz matrices}

Denote by $\mathbb{T}$ the unit circle in the complex plane and consider the sets

$$
\begin{aligned}
& \mathcal{W}=\left\{a(z): \mathbb{T} \rightarrow \mathbb{C}: \quad a(z)=\sum_{i \in \mathbb{Z}} a_{i} z^{i}, \sum_{i \in \mathbb{Z}}\left|a_{i}\right|<\infty\right\}, \\
& \mathcal{W}_{1}=\left\{a(z) \in \mathcal{W}: \quad a^{\prime}(z) \in \mathcal{W}\right\}, \quad a^{\prime}(z)=\sum_{i \in \mathbb{Z}} i a_{i} z^{i-1} .
\end{aligned}
$$

It is well known that $\mathcal{W}$ and $\mathcal{W}_{1}$ are Banach algebras with the norms $\|a\|_{\mathcal{W}}:=\sum_{i \in \mathbb{Z}}\left|a_{i}\right|$ and $\|a\|_{\mathcal{W}_{1}}:=\sum_{i \in \mathbb{Z}}\left|a_{i}\right|+\sum_{i \in \mathbb{Z}}\left|i a_{i}\right|$, respectively. That is, they are Banach spaces which are closed under multiplication and $\|a b\| \leq\|a\| \cdot\|b\|$ for $\|\cdot\|$ being either $\|\cdot\|_{\mathcal{W}}$ or $\|\cdot\|_{\mathcal{W}_{1}}$.

We associate with $a(z) \in \mathcal{W}$ the infinite Toeplitz matrix $T(a)=\left(t_{i, j}\right)$ such that $t_{i, j}=a_{j-i}$, $i, j \in \mathbb{Z}^{+}$.

It is well known that the set of matrices of the kind $T(a)+K$ where $a(z)$ is a continuous function and $K$ is a compact operator is a Banach algebra of operators in $\ell^{2}$ [12, Example 2.28]. Namely, it is a Banach space closed under multiplication, i.e., if $A=T(a)+K_{a}$ and $B=T(b)+K_{b}$, then $C=A B$ is such that $C=T(c)+K_{c}$ for some continuous function $c(z)$ and compact operator $K_{c}$.

For computational reasons, in order to keep under control the norm of the Toeplitz part $T(a)$ and the norm of the compact part $K_{a}$, a slightly different set with a different norm has been introduced in [8]. Define the class

$$
\mathcal{F}=\left\{F=\left(f_{i, j}\right): \quad \sum_{i, j \in \mathbb{Z}^{+}}\left|f_{i, j}\right|<\infty\right\},
$$

equipped with the norm $\|F\|_{\mathcal{F}}=\sum_{i, j \in \mathbb{Z}^{+}}\left|f_{i, j}\right|$ and consider the set of quasi-Toeplitz matrices defined as

$$
\mathcal{Q T}=\left\{A=T(a)+E: \quad a(z) \in \mathcal{W}_{1}, E \in \mathcal{F}\right\} .
$$

It has been shown in [8] that $\mathcal{Q T}$ is a Banach algebra with the norm $\|A\|=\|a\|_{\mathcal{W}_{1}}+\|E\|_{\mathcal{F}}$. The class $\mathcal{Q T}$ can be viewed as the computational counterpart of the more general case where $A$ is an operator in $\ell^{2}, a(z)$ is continuous and $E$ is compact.

Due to the decay of the moduli of the coefficients $a_{i}$ of any function $a(z) \in \mathcal{W}_{1}$ and to the decay of the moduli of the entries $f_{i, j}$ of any matrix $F \in \mathcal{F}$, it is possible to represent, up to any given precision, the matrix $A=T(a)+F \in \mathcal{Q T}$ with a finite number of parameters. More specifically, $a(z)$ is approximated by the Laurent polynomial $\sum_{i=-h}^{k} a_{i} z^{i}$, for sufficiently large integers $h, k>0$ and the matrix $F$ can be approximated by its leading $m \times n$ principal submatrix $\widetilde{F}$ for sufficiently large integers $m, n>0$. A further optimization of this representation is obtained by representing the latter $m \times n$ matrix $\widetilde{F}$ by means of the product $\widetilde{F}=U V^{T}$ where the matrices $U$ and $V$ have a number $k$ of columns given by the numerical rank of $\widetilde{F}$ which generally is much lower than $m$ and $n$.

This way, we can introduce an approximate matrix arithmetic in the class $\mathcal{Q T}$. For more details, we refer the reader to [8] where the operations of addition, multiplication and inversion for 
matrices in $\mathcal{Q T}$ are introduced together with algorithms for their computation and for the efficient representation of the correction $F$.

Given positive numbers $r_{1}<r_{2}$ and $r$, we denote by

$$
\mathbb{A}\left(r_{1}, r_{2}\right)=\left\{z \in \mathbb{C}: \quad r_{1}<|z|<r_{2}\right\}, \quad \mathbb{B}(r)=\{z \in \mathbb{C}: \quad|z|<r\}
$$

the annulus with radii $r_{1}$ and $r_{2}$ centered at 0 , and the disc of radius $r$ centered in 0 . If $r_{1}<1<r_{2}$ and if the symbol $a(z)$ is analytic over $\mathbb{A}\left(r_{1}, r_{2}\right)$, then it is well known that the coefficients $a_{i}$ decay exponentially to zero. This fact makes it easier to approximate $a(z)$ by means of a Laurent polynomial.

\subsection{Cyclic reduction}

In this section we assume that $A_{i}, i=-1,0,1$, are matrices of finite size. One of the most reliable and fast algorithms for computing the solutions $R$ and $G$ of equations (3) and (4), is Cyclic Reduction (CR) $[7,6,13,14]$ which is based on generating the following matrix sequences

$$
\begin{aligned}
& A_{0}^{(h+1)}=A_{0}^{(h)}+A_{1}^{(h)} S^{(h)} A_{-1}^{(h)}+A_{-1}^{(h)} S^{(h)} A_{1}^{(h)}, \quad S^{(h)}=\left(I-A_{0}^{(h)}\right)^{-1}, \\
& A_{1}^{(h+1)}=A_{1}^{(h)} S^{(h)} A_{1}^{(h)}, \quad A_{-1}^{(h+1)}=A_{-1}^{(h)} S^{(h)} A_{-1}^{(h)}, \\
& \widehat{A}^{(h+1)}=\widehat{A}^{(h)}+A_{-1}^{(h)} S^{(h)} A_{1}^{(h)}, \\
& \widetilde{A}^{(h+1)}=\widetilde{A}^{(h)}+A_{1}^{(h)} S^{(h)} A_{-1}^{(h)}, \quad h=0,1, \ldots
\end{aligned}
$$

with $A_{0}^{(0)}=\widetilde{A}^{(0)}=\widehat{A}^{(0)}=A_{0}, A_{1}^{(0)}=A_{1}, A_{-1}^{(0)}=A_{-1}$. Equations (8) can be applied if the matrix $S^{(h)}$ is invertible at any step of CR. Otherwise a breakdown is encountered.

Let us introduce the Laurent matrix polynomials $\varphi(z)=A_{-1} z^{-1}+A_{0}-I+A_{1} z$ and $\varphi^{(h)}(z)=$ $A_{-1}^{(h)} z^{-1}+A_{0}^{(h)}-I+A_{1}^{(h)} z$, and their formal inverses $\psi(z)=\varphi(z)^{-1}, \psi^{(h)}(z)=\varphi^{(h)}(z)^{-1}$.

The following properties hold, see [7].

\section{Theorem 2}

Assume that the matrix function $\varphi(z)=z^{-1} A_{-1}+\left(A_{0}-I\right)+z A_{1}$ is invertible for $z \in \mathbb{A}\left(r_{1}, r_{2}\right)$, where $r_{1}<1<r_{2}$. Let $\psi(z)=\varphi(z)^{-1}=\sum_{i=-\infty}^{+\infty} z^{i} H_{i}$ for $z \in \mathbb{A}\left(r_{1}, r_{2}\right)$. Assume that CR can be carried out with no breakdown. Then

1. $\psi^{(h)}(z)=\sum_{j \in \mathbb{Z}} z^{j} H_{j \cdot 2^{h}}$, moreover, $\varphi^{(h)}(z)$ and $\psi^{(h)}(z)$ are analytic for $z \in \mathcal{A}\left(r_{1}^{2^{h}}, r_{2}^{2^{h}}\right)$;

2. if $\operatorname{det} H_{0} \neq 0$ then there exists $h_{0}>0$ such that for any $h \geq h_{0}$ the matrix function $\psi^{(h)}(z)$ is nonsingular in $\mathcal{A}$ and the sequence $\varphi^{(h)}(z)$ converges to $H_{0}^{-1}$ uniformly over all the compact sets $\mathcal{K} \subset \mathbb{A}\left(r_{1}, r_{2}\right)$; moreover, for any matrix norm $\|\cdot\|$ and for any $\epsilon$ there exist a positive constant $\theta$ such that

$$
\begin{aligned}
& \left\|A_{-1}^{(h)}\right\| \leq \theta\left(r_{1}+\epsilon\right)^{2^{h}}, \quad\left\|A_{1}^{(h)}\right\| \leq \theta /\left(r_{2}-\epsilon\right)^{2^{h}}, \\
& \left\|A_{0}^{(h)}-H_{0}^{-1}\right\| \leq \theta\left(\frac{r_{1}+\epsilon}{r_{2}-\epsilon}\right)^{2^{h}} .
\end{aligned}
$$

If the sequences $\left(I-\widehat{A}^{(h)}\right)^{-1}$ and $\left(I-\widetilde{A}^{(h)}\right)^{-1}$ are uniformly bounded, then the sequence $A_{1}\left(I-\widetilde{A}^{(h)}\right)^{-1}$ converges to the solution $R$ of the matrix equation (3) of minimal spectral radius, while the sequence $\left(I-\widehat{A}^{(h)}\right)^{-1} A_{-1}$ converges to solution $G$ of the matrix equation (4) with minimal spectral radius.

In Theorem 2 the matrix function $\varphi(z)$ is assumed to be invertible in an annulus containing the unit circle. This condition can be weakened, by assuming that the function $\varphi(t z)$ is invertible in an annulus containing the unit circle, for a suitable scalar $t$ (see [7]). Indeed, in the case where $A_{-1}$, $A_{0}$ and $A_{1}$ define the Toeplitz part of the transition matrix of a QBD process, the function $\varphi(z)$ is not invertible for $z=1$. However, if the QBD is not null recurrent, we may find a scalar $t$ such that $\varphi(t z)$ is invertible in an annulus containing the unit circle, so that the convergence properties of CR stated by Theorem 2 hold. 
If the matrix $A_{0}^{(h)}-I$ is not invertible, then CR encounters a breakdown at step $h$. However, for problems coming from stochastic processes, like QBDs, where the matrix $P$ in (1) is stochastic and irreducible, the matrix $A_{0}^{(h)}-I$ is invertible for any $h$, so that breakdown is not encountered.

\section{FACTORIZATIONS AND QUADRATIC MATRIX EQUATIONS}

In this section, we assume that the matrices $A_{-1}, A_{0}$ and $A_{1}$ belong to a Banach algebra $\mathcal{B}$ with norm $\|\cdot\|$ and with identity $I$. We extend some results valid in the finite-dimensional case, which relate solutions $R, G$ of equations (3) and (4), respectively, to the Wiener-Hopf factorization of the Laurent matrix polynomial $\varphi(z):=z^{-1} A_{-1}+\left(A_{0}-I\right)+z A_{1}$.

In the finite case, this factorization plays a fundamental role for analyzing both the convergence of the CR and the properties of $R$ and $G$. We refer the reader to Chapter 3 of the book [7] for more details on this regard.

This generalization to coefficients belonging to a Banach algebra is motivated by the fact that, for QBDs with an infinite number of phases, the matrix $P$ has blocks which define operators in $L_{\infty}$, moreover, also the minimal nonnegative solutions $R$ and $G$ of the matrix equations (3), (4) belong to $L_{\infty}$. In particular, the existing results valid in the finite dimensional case might not hold.

We recall that the spectrum $\Lambda_{A}$ of $A \in \mathcal{B}$ is given by the set $\Lambda_{A}=\{z \in \mathbb{C}: \quad z I-$ $A$ is not invertible in $\mathcal{B}\}$. It is well known, see [12, pag. 2-3], that $\Lambda_{A}$ is a closed subset of $\mathbb{C}$. The real number $\delta(A)=\sup _{\lambda \in \Lambda}|\lambda|$, called the spectral radius of $A$, is such that $\lim _{k \rightarrow \infty}\left\|A^{k}\right\|^{\frac{1}{k}}=\delta(A)$, moreover, $\left\|A^{k}\right\|^{\frac{1}{k}} \leq\|A\|$ so that $\delta(A) \leq\|A\|$. We recall also that, if $\delta(A)<1$ then $I-A$ is invertible and $(I-A)^{-1}=\sum_{i=0}^{\infty} A^{i}$.

\section{Remark 1}

From the limit property of $\left\|A^{k}\right\| \frac{1}{k}$ it follows that for any $\epsilon>0$ there exists an integer $N>0$ such that for any $k \geq N$ it holds $\left\|A^{k}\right\|^{\frac{1}{k}}<\delta(A)+\epsilon$. In particular, if $\delta(A)<1$ and if $\epsilon>0$ is such that $\delta(A)+\epsilon<1$, then $\left\|A^{k}\right\|<(\delta(A)+\epsilon)^{k}<1$. Thus, if $k \geq N, k=q N+r$, where $q$ and $r$ are quotient and remainder of the division of $k$ by $N$, one finds that $\left\|A^{k}\right\| \leq\left\|A^{r}\right\| \cdot\left\|A^{N}\right\|^{q}$. This implies that if $\delta(A)<1$ then $\lim _{k \rightarrow \infty}\left\|A^{k}\right\|=0$.

The following result provides bound on the decay of the matrix coefficient of the inverse of a Laurent matrix polynomial.

\section{Lemma 1}

Let $A_{i} \in \mathcal{B} i=-1,0,1$ and assume that $\varphi(z)=A_{-1} z^{-1}+\left(A_{0}-I\right)+A_{1} z$ is invertible for $z \in$ $\mathbb{A}\left(t^{-1}, t\right)$ for a given $t>1$. Then $\psi(z)=\varphi(z)^{-1}=\sum_{j \in \mathbb{Z}} z^{j} H_{j}$ with $H_{j} \in \mathcal{B}$. Moreover, for any $t^{-1}<\sigma<t$ we have

$$
\left\|H_{j}\right\| \leq M(\sigma) \cdot \sigma^{-j}, \quad M(\sigma):=\max _{z \in \mathbb{T}}\|\psi(z \sigma)\| .
$$

Proof

Let $\sigma$ be such that $t^{-1}<\sigma<t$. Notice that, since $\varphi(z)$ is invertible for $|z|=\sigma$, then $\|\psi(z)\|$ depends continuously on $z$, so that the value $M(\sigma)$ is well-defined and finite. From the integral representation of the coefficients of a Laurent series [15], we have the relation

$$
H_{j}=\frac{1}{2 \pi \mathbf{i}} \int_{|z|=\sigma} z^{-(j+1)} \psi(z) d z
$$

where the integral is meant componentwise. Applying the norm on both sides yields

$$
\left\|H_{j}\right\| \leq \frac{1}{2 \pi} \int_{|z|=\sigma}\|\psi(z)\| \sigma^{-(j+1)} d z \leq M(\sigma) \cdot \sigma^{-j} .
$$


The existence of solutions $R$ and $G$ of equations (3) and (4) having disjoint spectra is equivalent to saying that there exists the factorization

$$
\varphi(z)=(I-z R) \cdot W \cdot\left(I-z^{-1} G\right) .
$$

We refer the reader to [16, Section 22] for this property in the case of operator polynomials. The next result provides a correspondence between the solutions $G, R$ and the coefficients of the inverse of $\varphi(z)$. In particular, this result extends Theorem 3.20 of [7] to the infinite dimensional case.

\section{Theorem 3}

Let $\varphi(z)=z^{-1} A_{-1}+\left(A_{0}-I\right)+z A_{1}$, where $A_{i} \in \mathcal{B}$ for $i=-1,0,1$. Assume that there exist $R, G \in \mathcal{B}$ which solve (3) and (4), respectively, having disjoint spectra, or equivalently, that

$$
\varphi(z)=(I-z R) \cdot W \cdot\left(I-z^{-1} G\right),
$$

for some invertible $W \in \mathcal{B}$. Moreover, assume that $\Lambda_{G} \subset \mathbb{B}\left(t^{-1}\right)$ and $\Lambda_{R} \subset \mathbb{B}\left(t^{-1}\right)$ for some $t>1$. Then $\varphi(z)$ is invertible for $z \in \mathbb{A}\left(t^{-1}, t\right)$. Moreover, $H_{0}=\sum_{j=0}^{\infty} G^{j} W^{-1} R^{j} \in \mathcal{B}$, and setting $H_{i}=H_{0} R^{i}$ for $i>0$ and $H_{i}=G^{-i} H_{0}$ for $i<0$, it follows that

$$
\psi(z):=\varphi(z)^{-1}=\sum_{i \in \mathbb{Z}} z^{i} H_{i}, \quad z \in \mathbb{A}\left(t^{-1}, t\right),
$$

with $H_{i} \in \mathcal{B}$. Finally, if $H_{0}$ is invertible, then $G=H_{-1} H_{0}^{-1}$ and $R=H_{0}^{-1} H_{1}$.

Proof

Since $\Lambda_{G} \subset \mathbb{B}\left(t^{-1}\right)$ and $\Lambda_{R} \subset \mathbb{B}\left(t^{-1}\right)$, then $\delta(z R), \delta(z G)<1$ for $z \in \mathbb{A}\left(t^{-1}, t\right)$ so that $I-z R$ and $I-z G$ are invertible and $(I-z R)^{-1}=\sum_{i=0}^{\infty} z^{i} R^{i},\left(I-z^{-1} G\right)^{-1}=\sum_{i=0}^{\infty} z^{-i} G^{i}$. Thus, from (9) we get

$$
\psi(z)=\left(I-z^{-1} G\right)^{-1} W^{-1}(I-z R)^{-1}=\left(\sum_{j=0}^{\infty} z^{-j} G^{j}\right) W^{-1}\left(\sum_{j=0}^{\infty} z^{j} R^{j}\right),
$$

for any $z \in \mathbb{A}\left(t^{-1}, t\right)$. This shows that $\varphi(z)$ is invertible. In view of Lemma 1 we may write $\psi(z)=\sum_{i \in \mathbb{Z}} z^{i} H_{i}$. By equating the terms in the same power of $z$ in (10), we find that the coefficients $H_{j}$ satisfy the following equations

$$
H_{0}=\sum_{j=0}^{\infty} G^{j} W^{-1} R^{j}, \quad H_{i}= \begin{cases}H_{0} R^{i} & i>0 \\ G^{-i} H_{0} & i<0 .\end{cases}
$$

It remains to prove that $H_{0}^{(n)}=\sum_{j=0}^{n} G^{j} W^{-1} R^{j}$ forms a Cauchy sequence so that there exists $\lim _{n} H_{0}^{(n)}=H_{0} \in \mathcal{B}$. In order to prove this, for $n>m$ we consider $H_{0}^{(n)}-H_{0}^{(m)}=$ $\sum_{j=m+1}^{n} G^{j} W^{-1} R^{j}=G^{m+1}\left(\sum_{j=0}^{n-m-1} G^{j} W^{-1} R^{j}\right) R^{m+1}$. So that

$$
\left\|H_{0}^{(n)}-H_{0}^{(m)}\right\| \leq\left\|G^{m+1}\right\| \cdot\|S\| \cdot\left\|R^{m+1}\right\|, \quad S=\sum_{j=0}^{n-m-1} G^{j} W^{-1} R^{j} .
$$

From Remark 1, for any $\epsilon>0$ such that $\lambda_{G}=\delta(G)+\epsilon<1$ and $\lambda_{R}=\delta(R)+\epsilon<1$, there exists $N>0$ such that for $k \geq N,\left\|G^{k}\right\| \leq\left\|G^{r}\right\| \cdot\left\|G^{N}\right\|^{q} \leq \lambda_{G}^{q},\left\|R^{k}\right\| \leq\left\|R^{r}\right\| \cdot\left\|R^{N}\right\|^{q} \leq \lambda_{R}^{q}$, where $q$ and $r$ are quotient and remainder, respectively, of the division of $k$ by $N$, i.e., $k=N q+r$. Thus,

$$
\left\|H_{0}^{(n)}-H_{0}^{(m)}\right\| \leq\|S\| \cdot\left\|G^{r}\right\| \cdot\left\|R^{r}\right\| \lambda_{G}^{q} \lambda_{R}^{q}
$$

and, since $\lambda_{G}, \lambda_{R}<1$, if $\|S\|$ is bounded from above by a constant independent of $n$ and $m$, it follows that the sequence $H_{0}^{(n)}$ is Cauchy. In order to prove the boundedness of $\|S\|$, it is sufficient 
to decompose $n-m+1$ as $n-m+1=\hat{q} N+\hat{r}$, where $\hat{q}$ and $\hat{r}$ are quotient and remainder of the division of $n-m+1$ by $N$, so that we have

$$
S=\sum_{k=0}^{\hat{q}-1} G^{k N} T R^{k N}+G^{q N} T_{r} R^{q N}, \quad T=\sum_{j=0}^{N-1} G^{j} W^{-1} R^{j}, \quad T_{r}=\sum_{j=0}^{r} G^{j} W^{-1} R^{j}
$$

whence the bound $\|S\| \leq \gamma \sum_{k=0}^{\hat{q}} \lambda_{G}^{k} \lambda_{R}^{k}$, where $\gamma=\sum_{j=0}^{N-1}\left\|G^{j}\right\| \cdot\left\|W^{-1}\right\| \cdot\left\|R^{j}\right\|$ is a constant independent of $n$ and $m$. This completes the proof.

\section{Remark 2}

The hypotheses $\Lambda_{G}, \Lambda_{R} \subset B\left(t^{-1}\right)$ with $t>1$ can be relaxed to $\Lambda_{G} \subset B\left(r_{G}\right)$ and $\Lambda_{R} \subset B\left(r_{R}\right)$ with $r_{R}^{-1}>r_{G}$. In fact, under these assumptions $\varphi(z)$ is invertible in the annulus $\mathbb{A}\left(r_{G}, r_{R}^{-1}\right)$ and Theorem 3 applies to the rescaled Laurent polynomial $\varphi(\alpha z)$ with $\alpha=\sqrt{r_{G} r_{R}^{-1}}$ and matrices $\alpha^{-1} G, \alpha R$. Observing that, $\varphi(\alpha z)^{-1}=\sum_{j \in \mathbb{Z}} \alpha^{j} z^{j} H_{j}$, if $H_{0}$ is invertible then one finds

$$
\alpha^{-1} G=\alpha^{-1} H_{-1} H_{0}^{-1}, \quad \alpha R=H_{0}^{-1} \alpha H_{1},
$$

which gives us again $G=H_{-1} H_{0}^{-1}$ and $R=H_{0}^{-1} H_{1}$.

\section{CYCLIC REDUCTION IN A BANACH ALGEBRA}

In this section we study properties of $\mathrm{CR}$ when applied to matrices $A_{i}, i=-1,0,1$, that belong to a Banach algebra $\mathcal{B}$.

As in the finite dimensional case [6], CR can be expressed in functional form by means of the recursion

$$
\varphi^{(h+1)}\left(z^{2}\right)=\varphi^{(h)}(z)\left(I-A_{0}^{(h)}\right)^{-1} \varphi^{(h)}(-z), \quad h \geq 0,
$$

where $\varphi^{(h)}(z)=z^{-1} A_{-1}^{(h)}+\left(A_{0}^{(h)}-I\right)+z A_{1}^{(h)}$. Moreover, if $\varphi(z)$ is analytic and invertible in an annulus $\mathbb{A}\left(t^{-1}, t\right)$, for a suitable $t>1$, and if $\mathrm{CR}$ can be carried out without breakdown, then, by using an induction argument, as in the finite dimensional case (see [6]), it turns out that the function $\varphi^{(h)}(z)$ is invertible in the annulus $\mathbb{A}\left(t^{-2^{h}}, t^{2^{h}}\right)$ and

$$
\psi^{(h)}(z):=\varphi^{(h)}(z)^{-1}=\sum_{j \in \mathbb{Z}} z^{j} H_{2^{h} j} .
$$
2.

The next result provides conditions for convergence of cyclic reduction which extends Theorem

\section{Theorem 4}

Let $A_{i} \in \mathcal{B}, i=-1,0,1$. Assume that $\varphi(z)=z^{-1} A_{-1}+\left(A_{0}-I\right)+z A_{1}$ is invertible in $\mathbb{A}\left(t^{-1}, t\right)$ for a suitable $t>1$, denote $\psi(z)=\varphi(z)^{-1}=\sum_{i \in \mathbb{Z}} z^{i} H_{i}$ and assume that $H_{0}$ is invertible. If $\mathrm{CR}$ can be carried out without breakdown, then the sequences (8) are Cauchy sequences and $\lim _{h \rightarrow \infty} A_{-1}^{(h)}=\lim _{h \rightarrow \infty} A_{1}^{(h)}=0, \lim _{h \rightarrow \infty} A_{0}^{(h)}=I+H_{0}^{-1}$. Moreover, for any $1<\sigma<t$ there exists $\gamma>0$ such that $\left\|A_{-1}^{(h)}\right\| \leq \gamma \sigma^{-2^{h}},\left\|A_{1}^{(h)}\right\| \leq \gamma \sigma^{-2^{h}},\left\|A_{0}^{(h)}-I-H_{0}^{-1}\right\| \leq \gamma \sigma^{-2^{h+1}}$.

\section{Proof}

Equating the coefficients of the same degree in $z$ in equation

$$
\psi^{(h)}(z)\left(z^{-1} A_{-1}^{(h)}+\left(A_{0}^{(h)}-I\right)+z A_{1}^{(h)}\right)=I
$$

yields

$$
\left\{\begin{array}{l}
H_{0} A_{-1}^{(h)}+H_{-2^{h}}\left(A_{0}^{(h)}-I\right)+H_{-2^{h+1}} A_{1}^{(h)}=0 \\
H_{2^{h}} A_{-1}^{(h)}+H_{0}\left(A_{0}^{(h)}-I\right)+H_{-2^{h}} A_{1}^{(h)}=I \\
H_{2^{h+1}} A_{-1}^{(h)}+H_{2^{h}}\left(A_{0}^{(h)}-I\right)+H_{0} A_{1}^{(h)}=0 .
\end{array}\right.
$$


By proceeding as in the proof of Theorem 3 of [6], we get

$$
\begin{aligned}
& \left\|A_{-1}^{(h)}\right\| \leq\left\|H_{0}^{-1}\right\|^{2}\left\|H_{-2^{h}}\right\|+O\left(\left\|H_{-2^{h}}\right\|^{2}\right) \\
& \left\|A_{0}^{(h)}-I-H_{0}^{-1}\right\| \leq 2\left\|H_{0}^{-1}\right\|^{3}\left\|H_{-2^{h}}\right\|\left\|H_{2^{h}}\right\|+O\left(\left\|H_{-2^{h}}\right\|^{2}\left\|H_{2^{h}}\right\|^{2}\right) \\
& \left\|A_{1}^{(h)}\right\| \leq\left\|H_{0}^{-1}\right\|^{2}\left\|H_{2^{h}}\right\|+O\left(\left\|H_{2^{h}}\right\|^{2}\right) .
\end{aligned}
$$

This completes the proof in view of Lemma 1.

Concerning convergence to the matrices $R$ and $G$ we have the following result.

\section{Theorem 5}

Assume that the hypotheses of Theorem 4 hold and that there exist $R, G \in \mathcal{B}$ with $\Lambda_{G} \subset \mathbb{B}\left(t^{-1}\right)$ and $\Lambda_{R} \subset \mathbb{B}\left(t^{-1}\right)$, with $t>1$, such that (9) holds. If the sequences $\left\|\left(I-\widehat{A}^{(h)}\right)^{-1}\right\|$ and $\left\|\left(I-\widetilde{A}^{(h)}\right)^{-1}\right\|$ are uniformly bounded then $G=\lim _{h \rightarrow \infty}\left(I-\widehat{A}^{(h)}\right)^{-1} A_{-1}$ and $R=\lim _{h \rightarrow \infty} A_{1}\left(I-\widetilde{A}^{(h)}\right)^{-1}$. Moreover, for any $1<\sigma<t$ there exists $\gamma>0$ such that $\left\|G-\left(I-\widehat{A}^{(h)}\right)^{-1} A_{-1}\right\| \leq \gamma \sigma^{-2^{h+1}}$ and $\left\|R-A_{1}\left(I-\widetilde{A}^{(h)}\right)^{-1}\right\| \leq \gamma \sigma^{-2^{h+1}}$.

Proof

As in the finite dimensional case [6], we have $-A_{-1}+\left(I-\widehat{A}^{(h)}\right) G=A_{1}^{(h)} G^{2^{h}+1}$. Therefore, since $\left\|\left(I-\widehat{A}^{(h)}\right)^{-1}\right\|$ is uniformly bounded, we have $\left\|G-\left(I-\widehat{A}^{(h)}\right)^{-1} A_{-1}\right\| \leq \|(I-$ $\left.\widehat{A}^{(h)}\right)^{-1}\|\| A_{1}^{(h)}\|\| G^{2^{h}+1} \|$. Since $\delta(G)<1$ and $\lim _{h \rightarrow \infty} A_{1}^{(h)}=0$, then $\lim _{h \rightarrow \infty}\left\|A_{1}^{(h)} G^{2^{h}+1}\right\|=0$. The bound on the speed of convergence follows from Theorem 4 and Remark 1. A similar argument applies for $R$.

\section{THE CASE OF QBDS}

In this section we assume that $A_{i}, i=-1,0,1$ are nonnegative matrices such that $\left(A_{-1}+A_{0}+\right.$ $\left.A_{1}\right) \mathbf{1}=\mathbf{1}$, which belong to the Banach algebra $\mathcal{Q T}$, with the norm $\|A\|=\|a\|_{\mathcal{W}_{1}}+\|E\|_{\mathcal{F}}$. We also assume that the minimal nonnegative solutions $R$ and $G$ to (3) and (4), respectively, belong to $\mathcal{Q T}$ as well.

We restrict our analysis to the case where the associated QBD is positive recurrent. Moreover, we assume $c(R)>1$, where $c(R)$ is defined in (7). Therefore, according to the properties on QBDs reported in Section 2.1, there exists $0<\alpha<1$ such that the series $\sum_{i=0}^{\infty} \alpha^{-i} R^{i}$ and $\sum_{i=0}^{\infty} \alpha^{i} G^{i}$ are convergent elementwise. Here, we also make a slightly stronger assumption on the spectrum of $\alpha G$ and $\alpha^{-1} R$. More specifically, we assume that there exists $\alpha$, with $0<\alpha<1$, such that $\Lambda_{\alpha G} \subset \mathbb{B}\left(t^{-1}\right)$ and $\Lambda_{\alpha^{-1} R} \subset \mathbb{B}\left(t^{-1}\right)$, for a suitable $t>1$.

\subsection{Properties of the solutions of the quadratic equations}

Under our assumptions, in view of Remark 2, the minimal nonnegative solutions of (4) and (3) are of the form $G=T(g)+E_{g}$ and $R=T(r)+E_{r}$, with $g(z), r(z) \in \mathcal{W}_{1}$ and $E_{g}, E_{r} \in F$. Now, we try to retrieve some further information on the symbols $g(z)$ and $r(z)$ of such matrices. As pointed out in [8] when we use the $\mathrm{CR}$ in the case $A_{i} \in \mathcal{Q T}, i=-1,0,1$ all the matrices generated by $\mathrm{CR}$ belong to $\mathcal{Q} \mathcal{T}$. Moreover, the Toeplitz part of these matrices $A_{i}^{(h)}, i=-1,0,1, \widehat{A}^{(h)}$ and $\widetilde{A}^{(h)}$ have associated symbols $a_{i}^{(h)}(z), i=-1,0,1, \widehat{a}^{(h)}(z), \widetilde{a}^{(h)}(z)$, respectively, which satisfy the same recurrence equations as (8). More precisely we have the scalar functional relations

$$
\begin{aligned}
& a_{0}^{(h+1)}(z)=a_{0}^{(h)}(z)+2 a_{1}^{(h)}(z) a_{-1}^{(h)}(z) /\left(1-a_{0}^{(h)}(z)\right), \\
& a_{1}^{(h+1)}(z)=a_{1}^{(h)}(z)^{2} /\left(1-a_{0}^{(h)}(z)\right), \quad a_{-1}^{(h+1)}(z)=a_{-1}^{(h)}(z)^{2} /\left(1-a_{0}^{(h)}(z)\right), \\
& \widetilde{a}^{(h+1)}(z)=\widetilde{a}^{(h)}(z)+a_{1}^{(h)}(z) a_{-1}^{(h)}(z) /\left(1-a_{0}^{(h)}(z)\right),
\end{aligned}
$$


with $h=0,1, \ldots$, where $a_{i}^{(0)}(z)=a_{i}(z), i=-1,0,1$ and $\widetilde{a}^{(0)}(z)=a_{0}(z)$. Observe that since all the quantities in the above recurrence are scalar functions, they commute so that $\widehat{a}^{(h)}(z)$ coincides with $\widetilde{a}^{(h)}(z)$. Moreover, we may easily verify that $\widetilde{a}^{(h)}(z)=\frac{1}{2}\left(a_{0}^{(h)}(z)+a_{0}^{(0)}(z)\right)$ for any $h \geq 0$.

In the scalar case CR reduces to the Graeffe iteration — see [17] and [6] — whose properties have been investigated in [18]. In particular, we know that if, for a given $z \in \mathbb{T}$ the polynomial

$$
p_{z}(x):=a_{1}(z) x^{2}+\left(a_{0}(z)-1\right) x+a_{-1}(z)
$$

associated with the triple $\left(a_{-1}(z), a_{0}(z)-1, a_{1}(z)\right)$, has one root $g(z)$ inside the unit disk and one root $r(z)^{-1}$ outside, then the sequence $a_{-1}(z)\left(1-\widetilde{a}^{(h)}(z)\right)^{-1}$ tends to $g(z)$ while $a_{1}(z)(1-$ $\left.\widetilde{a}^{(h)}(z)\right)^{-1}$ tends to $r(z)$. Thus, under our assumptions, for Theorems 4 and 5 the functions $g(z)$ and $r(z)$ correspond to the symbols of the matrices $G$ and $R$ which are the minimal nonnegative solutions to (4) and (3), respectively.

In the case where $a_{i}(z)=a_{i,-1} z^{-1}+a_{i, 0}+a_{i, 1} z$, for $i=-1,0,1$, it has been shown in [8] that, if $a_{-1,0} \neq 0$ or $a_{1,0} \neq 0$, and if $a_{i, j} \neq 0$ for at least a pair $(i, j)$ with $j \neq 0$, then for any $z \in \mathbb{T}, z \neq 1$, the polynomial $p_{z}(x)$ of (11) has a root of modulus less than 1 and a root of modulus greater than 1 .

\subsection{Necessary conditions for $G \in \mathcal{Q T}$}

There are cases where the minimal nonnegative solutions $R$ and $G$ to equations (3) and (4) do not belong to $\mathcal{Q T}$. An example is reported below.

Let $Z$ be the down-shift matrix having ones in the lower diagonal and zeros elsewhere, let $e_{1}=(1,0,0, \ldots)^{T}$ and define $A_{-1}=e_{1} e_{1}^{T}, A_{0}=\frac{1}{2} Z, A_{1}=\frac{1}{2}\left(I-e_{1} e_{1}^{T}\right)$. Observe that the matrix $A_{-1}+A_{0}+A_{1}$ is stochastic, $A_{-1}, A_{0}, A_{1} \in \mathcal{Q T}$, and that equations (3) and (4), have minimal nonnegative solutions

$$
R=\frac{1}{2}\left[\begin{array}{c|c}
0 & 0 \\
\hline \mathbf{1} & \left(I-\frac{1}{2} Z\right)^{-1}
\end{array}\right], \quad G=\mathbf{1} e_{1}^{T},
$$

respectively, in particular, as expected, $G \mathbf{1}=\mathbf{1}$. On the other hand $G$ and $R$ do not belong to $\mathcal{Q T}$ since their corrections to the Toeplitz part are neither in $\mathcal{F}$ nor have a bounded 2-norm.

We present a necessary condition that has to be satisfied in order to guarantee that the solution $G$ of the matrix equation (4) belongs to $\mathcal{Q T}$.

Lemma 2

Under the assumptions of Theorem 1, let $\varphi(z)=z^{-1} A_{-1}+\left(A_{0}-I\right)+z A_{1}$ with $A_{i} \in \mathcal{Q T}$. Let $a_{i}(z)$ be the symbols associated with the blocks $A_{i}$, let $g(z)$ be the minimal non-negative Laurent series $^{\dagger}$ such that

$$
a_{-1}(z)+a_{0}(z) g(z)+a_{1}(z) g(z)^{2}=g(z), \quad g(z)=\sum_{i \in \mathbb{Z}} g_{i} z^{i},
$$

and assume that the $\mathrm{QBD}$ is positive recurrent. If the minimal non-negative solution $G$ of (4) belongs to $\mathcal{Q T}$ then $g(1)=1$.

\section{Proof}

Assume that $G=T(g)+E_{g} \in \mathcal{Q T}$, where $T(g)$ is the Toeplitz part and $E_{g} \in \mathcal{F}$. Since $G$ verifies (4) then the symbol of $T(g)$ needs to be $g(z)$. Since the QBD is positive recurrent, then $G \mathbf{1}=\mathbf{1}$ and we have $E_{g} \mathbf{1} \geq \mathbf{1}-T(g) \mathbf{1} \geq \epsilon \mathbf{1}$, with $\epsilon=1-g(1)$. If $\epsilon>0$ then every row of $E_{g}$ has sum of moduli at least $\epsilon$, and therefore $E_{g} \notin \mathcal{F}$, which leads to a contradiction.

In the example at the beginning of this section, the matrix $G$ is such that $g(1)=0$, therefore, for Lemma 2, $G$ cannot belong to $\mathcal{Q T}$.

\footnotetext{
${ }^{\dagger}$ we say that $a(z) \leq b(z)$ if the inequality holds coefficient-wise, and by $g(z)$ non-negative we mean that its coefficients are all non-negative. 


\section{Remark 3}

Note that the symbol $g(z)$ can be computed easily by applying the iterative scheme of cyclic reduction on the power series $a_{i}(z)$ [8]. This provides an effective necessary criterion in order that $G \in \mathcal{Q T}$, indeed, if $g(1)<1$ then $G$ cannot belong to $\mathcal{Q T}$.

\subsection{Handling problematic cases}

In Lemma 2 we highlighted a necessary condition in order that $G \in \mathcal{Q T}$. In some of the instances of the problems shown in the paper by Motyer and Taylor [9] this condition is not satisfied, and the sequences generated by cyclic reduction do not converge inside $\mathcal{Q T}$. However, it is possible to express the problem in a different equivalent form where this necessary condition is satisfied.

More precisely, the idea relies on the well-known permutation matrix $\Sigma$ that swaps the two factors of a Kronecker product, i.e., such that $\Sigma(A \otimes B) \Sigma^{T}=B \otimes A$. Since we are looking for a left eigenvector $\pi$ of $P$, we can instead compute the left eigenvector $\tilde{\pi}$ of $\Sigma P \Sigma^{T}$. It is immediate to note that $\pi=\tilde{\pi} \Sigma$.

Let us make the additional assumption that the blocks $A_{i}$ are tridiagonal, and have corrections with support in the first row. This is equivalent to asking that the matrix $P$ is of the following form

$$
P=\sum_{j=1}^{3} T_{j}^{(1)} \otimes T_{j}^{(2)}+\sum_{j=1}^{2} e_{1} u_{j}^{T} \otimes T_{j}^{(3)}+\sum_{j=1}^{2} T_{j}^{(4)} \otimes e_{1} v_{j}^{T},
$$

where the matrices $T_{j}^{(i)}$ are Toeplitz tridiagonal, and $u_{j}, v_{j}$ are either $e_{1}$ or $e_{2}$. The above structure is clearly invariant under the action of $\Sigma$, so that $\Sigma P \Sigma^{T}$ has the same structure of $P$. However, it can happen that the matrix equation associated with $\Sigma P \Sigma^{T}$ satisfies the criterion of Lemma 2, whilst $P$ does not. This is what happens in the cases 2, 6, and 10 from the paper [9].

In the numerical experiments, whenever the criterion of Lemma 2 was not satisfied, we applied this trick and we managed to compute $G, R$ and the invariant probability vector $\pi$.

\section{COMPUTING THE STEADY STATE VECTOR}

We now discuss the computation of the invariant vector $\pi$, that represents the steady state vector of the QBD process. As recalled in Section 2.1, we have

$$
\pi_{0}=\pi_{0}\left(B_{0}+B_{1} G\right), \quad \pi_{0}(I-R)^{-1} \mathbf{1}=1, \quad \pi_{n}=\pi_{n-1} R=\pi_{0} R^{n} .
$$

where $\pi=\left[\begin{array}{lll}\pi_{0} & \pi_{1} & \ldots\end{array}\right]$. This reduces the problem of computing $\pi$ to the computation of $R$ and $\pi_{0}$. Notice that both $\pi$ and $\pi_{0}$ are infinite vectors. In particular, $\pi_{0} \in \ell^{1}(\mathbb{N})$, so that, for any $\epsilon>0$, there exists an index after which all its entries are smaller than $\epsilon$ in magnitude. It would be unfeasible to compute an infinite number of entries on a computer, so we assume that a certain threshold $\epsilon>0$ has been fixed once and for all, and we are only interested in computing the components of $\pi$ of magnitude larger than $\epsilon$. In our experiments we always choose $\epsilon$ equal to the machine precision $\epsilon \approx 2.22 \cdot 10^{-16}$.

If the solutions $G$ and $R$ of the matrix equations in (3) are in $\mathcal{Q T}$, the infinite vectors $\pi_{n}$ can be easily computed. Let us consider the first block $\pi_{0}$. Since $G \in \mathcal{Q T}$, then $M:=B_{0}+B_{1} G \in \mathcal{Q T}$, so that it is numerically banded, and has a correction in the top-left corner. Using a suitable reblocking, one can interpret the matrix $M$ as numerically block tridiagonal and Toeplitz, with the only exception of the first block row. More precisely, by choosing sufficiently large blocks $\hat{M}_{i}, M_{i}$, we can rephrase the problem as follows:

$$
\left[\begin{array}{lll}
\pi_{0}^{(0)} & \pi_{0}^{(1)} & \ldots
\end{array}\right] \underbrace{\left[\begin{array}{ccccc}
\hat{M}_{0} & \hat{M}_{1} & & & \\
M_{-1} & M_{0} & M_{1} & & \\
& M_{-1} & M_{0} & M_{1} & \\
& & \ddots & \ddots & \ddots
\end{array}\right]}_{M}=\left[\begin{array}{llll}
\pi_{0}^{(0)} & \pi_{0}^{(1)} & \ldots
\end{array}\right] .
$$


The matrix $\hat{M}_{0}$ is chosen so that it contains the correction part of $M$, and the other blocks $M_{i}$ contain the banded Toeplitz part. More precisely, the size $m$ of the blocks $M_{i}$ is chosen as $m=\max \left\{b_{\ell}, b_{u}\right\}$ where $b_{\ell}, b_{u}$ are the lower and upper bandwidth of $M$ after the truncation with the relative threshold $\epsilon$. In particular, the matrix $M$ represents the transition matrix of a QBD with finite dimensional blocks. Since the original process is positive recurrent, then the QBD associated with the matrix $M$ can be seen as a "censored" version of the original one, that is still positive recurrent. Therefore, solving (12) consists in computing the steady state vector of a QBD, thus $\pi_{0}^{(0)}\left(\hat{M}_{0}+\hat{M}_{1} G_{M}\right)=\pi_{0}^{(0)}$ and $\pi_{0}^{(k)}=\pi_{0}^{(k-1)} R_{M}, k \geq 1$, where $R_{M}$ and $G_{M}$ are the minimal nonnegative solutions of the matrix equations:

$$
R_{M}^{2} M_{-1}+R_{M} M_{0}+M_{1}=R_{M}, \quad M_{-1}+M_{0} G_{M}+M_{1} G_{M}^{2}=G_{M},
$$

respectively. Since the spectral radius of $R_{M}$ is smaller than 1 , one can give explicit estimates of the number of non negligible components $\pi_{0}^{(k)}$ with respect to $\epsilon$. In our numerical experiments we stop when $\left\|\pi_{0}^{(k)}\right\|_{\infty}<\epsilon \cdot\left\|\pi_{0}^{(0)}\right\|_{\infty}$. For more details on this analysis we refer to [7].

Matrices $R_{M}$ and $G_{M}$ can be computed by applying cyclic reduction and the finite dimensional vector $\pi_{0}^{(0)}$ can be computed by applying a standard method for approximating the Perron vector of a nonnegative matrix.

Once $\pi_{0}$ is computed, the other entries can be recovered by right multiplication by $R$. These techniques will be used in the next section to solve some problems from a paper by Motyer and Taylor [9], which are representative examples of QBDs found in the applications.

\section{NUMERICAL RESULTS}

We tested some QBD processes, taken from [9], which describe two node Jackson tandem networks. We have computed the invariant probability vector $\pi$ using the strategy described in the previous section. The implementation of our tests and algorithms is available online at https://github. com/numpi/cqt-toolbox.

Recall that $\pi=\left[\begin{array}{lll}\pi_{0} & \pi_{1} & \ldots\end{array}\right]$, where each of the vectors $\pi_{i}$ has infinite entries which decay to 0 . Moreover, also the norms $\left\|\pi_{i}\right\|$ decay to 0 as $i \rightarrow \infty$. For the bidimensional nature of the problem it is convenient to represent $\pi$ as an infinite matrix by rearranging its entries as follows:

$$
M_{\pi}=\left[\begin{array}{c}
\pi_{0} \\
\pi_{1} \\
\vdots
\end{array}\right] .
$$

This has the advantage that the matrix has summable entries, and therefore we have $M_{\pi} \in \mathcal{Q T}$, with a null symbol. Moreover, this ordering is also meaningful from the point of view of the applications, since the states of the QBDs in [9] are naturally indexed as items of $\mathbb{N}^{2}$, and there is a one to one correspondence to this indexing and the entries of $M_{\pi}$.

We notice that the matrix $P$ with the blocks $\hat{A}_{i}, A_{i}$ can be written as

$$
P=I \otimes A_{0}+J \otimes A_{1}+J^{T} \otimes A_{-1}+e_{1} e_{1}^{T} \otimes\left(\hat{A}_{0}-A_{0}\right)+e_{1} e_{2}^{T}\left(\hat{A}_{1}-A_{1}\right),
$$

where $e_{j}$ is the vector with a 1 in position $j$, and $J$ is the matrix with 1 on the superdiagonal and zero elsewhere.

In particular, denoting $\operatorname{vec}(A)$ the vector obtained by stacking the columns of the matrix $A$, it is well known that $\operatorname{vec}(X)^{T} A \otimes B=\operatorname{vec}\left(A X^{T} B^{T}\right)^{T}$. This way, we can rephrase the condition that $\pi$ is a left eigenvector of the block tridiagonal matrix as $M_{\pi}$ being the solution of the following matrix equation:

$$
e_{1} e_{1}^{T} M_{\pi}\left(\hat{A}_{0}-A_{0}\right)+J M_{\pi} A_{-1}+J^{T} M_{\pi} A_{1}+M_{\pi} A_{0}=0
$$

where the term $e_{1} e_{2}^{T} M_{\pi} \hat{A}_{1}=A_{1}$ has been removed since for the Jackson tandem queues we have $\hat{A}_{1}=A_{1}$. Notice that all the matrices in the above equation are semi-infinite and belong to $\mathcal{Q T}$, so that we can easily verify the numerical validity of (13). 


\begin{tabular}{c|c|c|c} 
Problem & Residue $\|\cdot\|_{\mathcal{Q T}}$ & Residue $\|\cdot\|_{\infty}$ & Time $(\mathrm{s})$ \\
\hline 1 & $4.40 \mathrm{e}-13$ & $2.02 \mathrm{e}-13$ & 15.13 \\
$2^{*}$ & $1.94 \mathrm{e}-12$ & $9.09 \mathrm{e}-13$ & 1.14 \\
3 & $4.29 \mathrm{e}-13$ & $2.02 \mathrm{e}-13$ & 1.62 \\
4 & $4.08 \mathrm{e}-13$ & $1.77 \mathrm{e}-13$ & 10.29 \\
5 & $4.11 \mathrm{e}-13$ & $1.93 \mathrm{e}-13$ & 6.07 \\
$6^{*}$ & $2.48 \mathrm{e}-12$ & $1.16 \mathrm{e}-12$ & 4.23 \\
7 & $5.64 \mathrm{e}-13$ & $2.64 \mathrm{e}-13$ & 118.14 \\
8 & $8.80 \mathrm{e}-14$ & $4.30 \mathrm{e}-14$ & 2.68 \\
9 & $4.43 \mathrm{e}-13$ & $2.13 \mathrm{e}-13$ & 1.15 \\
$10^{*}$ & $1.38 \mathrm{e}-11$ & $6.65 \mathrm{e}-12$ & 1.05
\end{tabular}

Table I. Residue of the matrix equation (13) evaluated at the matrix $M_{\pi}$ computed numerically. The results are reported both in the $\mathcal{Q T}$ and in the $\infty$ norms, along with the time required to compute $\pi$. The problems marked with the symbol $*$ have been solved using the trick described in the section 5.3.

In Table I we have reported the residues of the evaluation of the matrix equation (13) at the solutions $M_{\pi}$ that we have computed numerically. We have tested all the problems that satisfy our hypotheses, so that cyclic reduction applied to the blocks $A_{i}$ converges in $\mathcal{Q T}$. In particular, problems 2,6 , and 10 do not have the required properties. In that cases, we have applied the strategy described in Section 5.3.

The residues are reported both in the $\mathcal{Q T}$ and in the $\infty$-norm, and we have reported the timings required to compute $\pi$ in Matlab on a laptop, with an Intel i5-6300U CPU.

\section{REFERENCES}

1. Jackson JR. Networks of waiting lines. Operations Research 1957; 5(4):518-521.

2. Miyazawa M. Light tail asymptotics in multidimensional reflecting processes for queueing networks. Top 2011; 19(2):233-299.

3. Kobayashi M, Miyazawa M. Revisiting the tail asymptotics of the double QBD process: refinement and complete solutions for the coordinate and diagonal directions. Matrix-analytic methods in stochastic models, Springer Proc. Math. Stat., vol. 27. Springer, New York, 2013; 145-185.

4. Latouche G, Ramaswami V. Introduction to Matrix Analytic Methods in Stochastic Modeling. ASA-SIAM Series on Statistics and Applied Probability, SIAM: Philadelphia PA, 1999.

5. Latouche G, Mahmoodi S, Taylor P. Level-phase independent stationary distributions for GI/M/1-type Markov chains with infinitely-many phases. Performance Evaluation 2013; 70(9):551-563.

6. Bini DA, Meini B. The cyclic reduction algorithm: from Poisson equation to stochastic processes and beyond. Numerical Algorithms 2009; 51(1):23-60.

7. Bini DA, Latouche G, Meini B. Numerical methods for structured Markov chains. Oxford University Press, 2005.

8. Bini DA, Massei S, Meini B. Semi-infinite quasi-Toeplitz matrices with applications to QBD stochastic processes. Mathematics of Computation 2017; Accepted for publication, arXiv:1611.06337.

9. Motyer AJ, Taylor PG. Decay rates for quasi-birth-and-death processes with countably many phases and tridiagonal block generators. Adv. Appl. Prob. 2006; 38:522-544.

10. Miyazawa M, Zhao YQ. The stationary tail asymptotics in the $G I / G / 1$-type queue with countably many background states. Adv. in Appl. Probab. 2004; 36(4):1231-1251.

11. Tweedie RL. Operator-geometric stationary distributions for Markov chains, with application to queueing models. Adv. in Appl. Probab. 1982; 14(2):368-391.

12. Böttcher A, Silbermann B. Introduction to large truncated Toeplitz matrices. Springer Science \& Business Media, 2012.

13. Buzbee BL, Golub GH, Nielson CW. On direct methods for solving Poisson's equations. SIAM Journal on Numerical Analysis 1970; 7(4):627-656.

14. Hockney RW. A fast direct solution of Poisson's equation using Fourier analysis. Journal of the ACM (JACM) 1965; 12(1):95-113.

15. Henrici P. Applied and computational complex analysis, discrete Fourier analysis, Cauchy integrals, construction of conformal maps, univalent functions, vol. 3. John Wiley \& Sons, 1993.

16. Markus AS. Introduction to the spectral theory of polynomial operator pencils, Translations of Mathematical Monographs, vol. 71. American Mathematical Society, Providence, RI, 1988.

17. Bini DA, Gemignani L, Meini B. Computations with infinite Toeplitz matrices and polynomials. Linear Algebra Appl. 2002; 343/344:21-61.

18. Ostrowski A. Recherches sur la méthode de Graeffe et les zéros des polynomes et des séries de Laurent. Acta Math. 1940; 72:99-155. 